\title{
Tratamento cirúrgico de fratura do complexo zigomático-orbital em paciente vítima de acidente desportivo: relato de caso
}

Surgical treatment of orbitozygomatic complex fracture in patient victims of sport accident: case report Tratamiento quirúrgico de la fractura compleja orbito-cigomática en pacientes víctimas de accidente deportivo: reporte de caso

José Jhenikártery Maia de OLIVEIRA ${ }^{1}$ Micaella Fernandes FARIAS ${ }^{1}$

Flaviana Laís Pereira dos SANTOS ${ }^{1}$

Artemisa Fernanda Moura FERREIRA ${ }^{2}$

Lucas Alexandre de Morais SANTOS ${ }^{3}$

${ }^{I}$ Centro Universitário João Pessoa - Unipê 58053-000 João Pessoa - PB, Brasil

${ }^{2}$ Doutoranda em Clínicas Odontológicas pela Universidade Federal da Paraíba, UFPB;

Mestre em Clínica Odontológica Integrada pela Universidade Federal de Pernambuco, UFPE

Professora do Curso de Odontologia do Centro Universitário de João Pessoa, UNIPE

${ }^{3}$ Doutorando e Mestre em Cirurgia e Traumatologia Bucomaxilofacial pela Faculdade de Odontologia da Universidade de Pernambuco, FOP-UPE. Professor do Curso de Odontologia do Centro Universitário de João Pessoa, UNIPÊ

\section{Resumo}

Introdução: Fraturas no complexo zigomático orbital podem resultar em deslocamento ósseo com alteração do volume orbital e distúrbios funcionais. As complicações associadas aos traumas dessa região podem vir a interferir diretamente na qualidade de vida do indivíduo, dessa forma, faz-se necessário a cirurgia de reposicionamento anatômico do osso. Objetivo: Relatar um caso clínico de fratura do complexo zigomático orbital em paciente vítima de acidente desportivo. Relato de caso: Paciente do sexo masculino, leucoderma, vítima de acidente desportivo, compareceu a um serviço de Cirurgia Bucomaxilofacial da cidade de Recife-PE, apresentando alterações anatômicas visíveis na região zigomática do lado esquerdo da face. Ao exame clínico observou-se assimetria facial, edema, equimose periorbital, hemorragia subconjuntival, perda de volume do lado afetado e desconforto relado pelo paciente, sugerindo fratura do complexo zigomático orbital. A Tomografia Computadorizada mostrou perda da projeção anteroposterior do corpo do osso zigomático esquerdo, com fratura no arco zigomático e descontinuidade da sutura esfenozigomática esquerdas. O corte tomográfico coronal evidenciou aprisionamento do conteúdo orbital por fragmentos presentes no assoalho da órbita, e fratura do pilar zigomático-maxilar. Através de acessos cirúrgicos peri-orbitais (superciliar e subtarsal) foi instalada 1 mini-placa com parafusos ( 1 em cada acesso), nas regiões da sutura fronto-zigomática e da margem infraorbital. Pelo acesso intraoral foi fixada uma placa no pilar zigomático-maxilar. O tratamento cirúrgico buscou acesso ao esqueleto da órbita, redução anatômica e fixação interna estável com mini-placas e parafusos. Considerações finais: Após 10 dias, observou-se o restabelecimento anatômico da projeção anteroposterior do osso zigomático direto, procedendo à melhora estético-funcional.

Descritores: Anatomia Regional; Traumatologia; Fraturas Orbitárias.

\section{Abstract}

Introduction: Fractures in the complex zygomatic orbital can result in bone dislocation with alteration of the orbital volume and altered disorders. Complications associated with traumas in this region may directly interfere with the individual's quality of life, thus, it is necessary to perform anatomical bone repositioning surgery. Objective: to report a clinical case of fracture of the orbital zygomatic complex in a patient victim of a sports accident. Case report: Male patient, leukoderma, victim of a sports accident, attended a service of Maxillofacial Surgery in the city of Recife-PE, anatomical changes to the zygomatic region of the left side of the face. Clinical examination revealed facial asymmetry, edema, periorbital ecchymosis, subconjunctival hemorrhage, loss of volume on the affected side and discomfort reported by the patient, suggesting fracture of the orbital zygomatic complex. Computed Tomography discovered the loss of the anteroposterior projection of the body of the left zygomatic bone, with fracture in the zygomatic arch and discontinuity of the left sphenozygomatic suture. The coronal tomographic section showed imprisonment of the orbital content by fragments present in the outbreak of the orbit, and fracture of the zygomatic-maxillary pillar. Through peri-orbital surgical accesses (superciliary and subtarsal), a mini-plate with screws (1 in each access) was installed in the regions of the fronto-zygomatic suture and the infraorbital margin. Through the intraoral access, a plate was fixed on the zygomatic-maxillary abutment. Surgical treatment sought access to the orbit skeleton, anatomical reduction and stable internal correction with mini-plates and screws. Final considerations: After 10 days, the anatomical restoration of the anteroposterior projection of the direct zygomatic bone was observed, proceeding to aesthetic-functional improvement.

Descriptores: Anatomy, Regional; Traumatology; Orbital Fractures.

\section{Resumen}

Introducción: Las fracturas en el orbitario cigomático complejo pueden resultar en una luxación ósea con alteración del volumen orbitario y alteraciones de los trastornos. Las complicaciones asociadas a los traumatismos en esta región pueden interferir directamente con la calidad de vida del individuo, por lo que es necesario realizar una cirugía de reposicionamiento óseo anatómico. Objetivo: reportar un caso clínico de fractura del complejo cigomático orbitario en un paciente víctima de un accidente deportivo. Caso clínico: Paciente de sexo masculino, leucodermia, víctima de accidente deportivo, acudió a un servicio de Cirugía Maxilofacial de la ciudad de Recife-PE, alteraciones anatómicas en la región cigomática del lado izquierdo de la cara. El examen clínico reveló asimetría facial, edema, equimosis periorbitaria, hemorragia subconjuntival, pérdida de volumen en el lado afectado y malestar referido por el paciente, sugestivo de fractura del complejo cigomático orbitario. La tomografía computarizada descubrió la pérdida de la proyección anteroposterior del cuerpo del hueso cigomático izquierdo, con fractura del arco cigomático y discontinuidad de la sutura esfenocigomática izquierda. El corte tomográfico coronal mostró aprisionamiento del contenido orbitario por fragmentos presentes en el brote de la órbita y fractura del pilar cigomático-maxilar. Mediante accesos quirúrgicos periorbitarios (superciliar y subtarsal) se instaló una miniplaca con tornillos (1 en cada acceso) en las regiones de la sutura fronto-cigomática y el margen infraorbitario. A través del acceso intraoral se fijó una placa sobre el pilar cigomático-maxilar. El tratamiento quirúrgico buscó acceso al esqueleto orbital, reducción anatómica y corrección interna estable con miniplacas y tornillos. Consideraciones finales: A los 10 días se observó la restauración anatómica de la proyección anteroposterior del hueso cigomático directo, procediendo a una mejoría estético-funcional.

Descriptores: Anatomía Regional; Traumatología; Fracturas Orbitales.

\section{INTRODUÇÃO}

O trauma maxilofacial é um problema de saúde pública relevante na sociedade atual. O mesmo representa uma das principais causas de morte e morbidade no mundo ${ }^{1}$, podendo resultar em grandes transtornos emocionais, funcionais e estéticos, interferindo diretamente na qualidade de vida do 
indivíduo vitimado. Embora apresente etiologia multifatorial, grande parte dos traumatismos maxilofaciais são decorrentes de acidentes de trânsito, agressão física e prática desportiva ${ }^{2-4}$.

A abordagem da condição traumática deve ser de abrangência multidisciplinar, envolvendo principalmente as especialidades da área, oftalmologia, cirurgia plástica, maxilofacial e neurocirurgia $^{5}$, objetivando evitar sequelas de um diagnóstico e tratamento inadequado.

O osso zigomático é uma das estruturas mais proeminentes da face, tornando-se vulnerável a injúrias traumáticas com maior frequência; participa da constituição das paredes lateral e inferior da órbita, bem como das paredes do osso temporal e fossa infratemporal, devido a relação articular deste osso com os ossos temporais, maxilares, frontal e asas maiores do esfenoide, portanto, fraturas nesta região podem provocar deslocamento ósseo com alteração do volume orbital ${ }^{6}$.

As fraturas do complexo zigomático se classificam com base nos achados clinicos visualizados na tomada radiográfica póstero-anterior de Walter. Para tal, esta classificação foi divida em seis grupos, onde o grupo I, não apresenta descolamento significativo; grupo II é constituido por fraturas da arcada zigomática, sem envolvimento das paredes do seio maxilar e órbita, associa-se esta condição ao trismo; grupo III fratura do corpo sem rotação, onde o paciente apresenta achatamento na bochecha e degrau na margem infraorbitária; grupo IV, apresenta fraturas do corpo zigomático com rotação medial; grupo $\mathrm{V}$, fratura do corpo rotacionadas lateralmente; grupo IV, fundamenta-se em fraturas complexas, abrangendo os casos em que as linhas de fratura no segmento principal são observadas ${ }^{6}$.

Dentre os sinais e sintomas encontrados na literatura frente à fratura do complexo zigomático orbital, cita-se: assimetria facial, sintomatologia dolorosa, parestesia do nervo infraorbital, trismo, equimose subconjuntival, restrição dos movimentos mandibulares e oculares, distopia, diplopia, proptose e alterações visuais significativas ${ }^{7}$, o que pode interferir diretamente na qualidade de vida.

O diagnóstico fundamenta-se na história abordando o tipo de trauma, intensidade e o tempo decorrido, visto que este último possui influência no tratamento a ser adotado, além da realização de exames físicos e solicitação de exames de imagem. A Tomografia Computadorizada proporciona a avaliação mais criteriosa das fraturas a fim do estabelecimento do diagnóstico e tratamento eficaz pela sua reconstrução tridimensional, sendo assim, trata-se da técnica mais utilizada frente a fraturas do terço médio da face ${ }^{6,8}$.

Este trabalho objetiva relatar um caso sobre fratura de complexo zigomático orbital tratada cirurgicamente por acesso ao esqueleto da órbita, redução anatômica e fixação interna estável com miniplacas e parafusos, considerando a importância do diagnóstico conciso a fim de determinar o tratamento adequado visando diminuir as complicações e sequelas da devida condição.

CASO CLÍNICO

Paciente do sexo masculino, leucoderma, vítima de acidente desportivo, compareceu ao serviço de Cirurgia Bucomaxilofacial do Hospital da Restauração Governador Paulo Guerra da cidade de Recife-PE, Brasil, apresentando alterações anatômicas visíveis na região zigomática do lado esquerdo da face. Ao exame clínico, observou-se assimetria facial, edema, equimose periorbital, hemorragia subconjuntival, perda de volume do complexo zigomático orbital do lado esquerdo da face (Figura 1) e desconforto relatado pelo paciente, sugerindo fratura do complexo zigomático orbital, confirmado após a aquisição das imagens tomográficas.

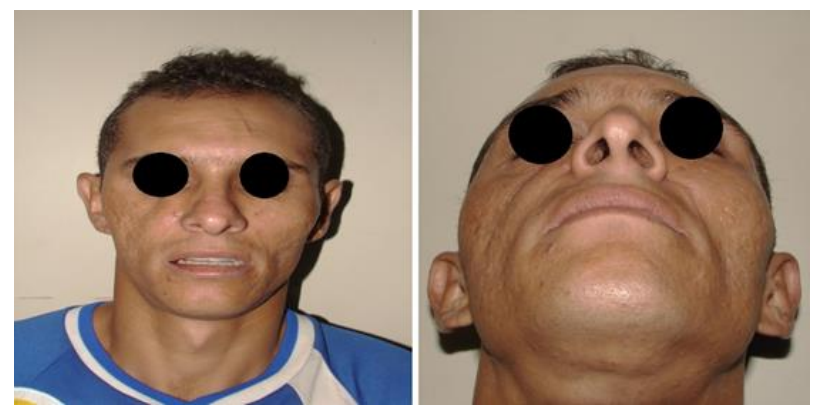

Figura 1: Assimetria facial com perda da projeção anteroposterior do complexo zigomático orbital esquerdo.

O diagnóstico definitivo foi estabelecido após a aquisição de Tomografia Computadorizada, que mostrou perda da projeção anteroposterior do corpo do osso zigomático direto, com fratura no arco zigomático e descontinuidade da sutura esfenozigomática. O corte tomográfico coronal evidenciou aprisionamento do conteúdo orbital por fragmentos presentes no assoalho da órbita, e fratura do pilar zigomático-maxilar (Figura 2). Diante disso, o paciente foi submetido ao tratamento cirúrgico, sob anestesia geral, para redução da fratura do osso zigomático e restabelecimento anatômico da cavidade orbital. O tratamento cirúrgico buscou acesso ao esqueleto da órbita, redução anatômica e fixação interna estável com mini-placas e parafusos.

Através de acessos cirúrgicos periorbitais (superciliar e subtarsal) (Figura 3) foi instalada miniplaca com parafusos (uma em cada acesso), nas regiões da sutura frontozigomática e da margem infraorbital (Figura 4), como também, foi instalada uma placa no pilar zigomático-maxilar pelo acesso intra-oral (Figura 5). Mediante o exposto, após 10 dias da terapia cirúrgica, o paciente retornou para avaliação clínica e topográfica, onde foi possível observar o restabelecimento anatômico da projeção 
anteroposterior do osso zigomático, acarretando em melhora estético-funcional (Figura 6).

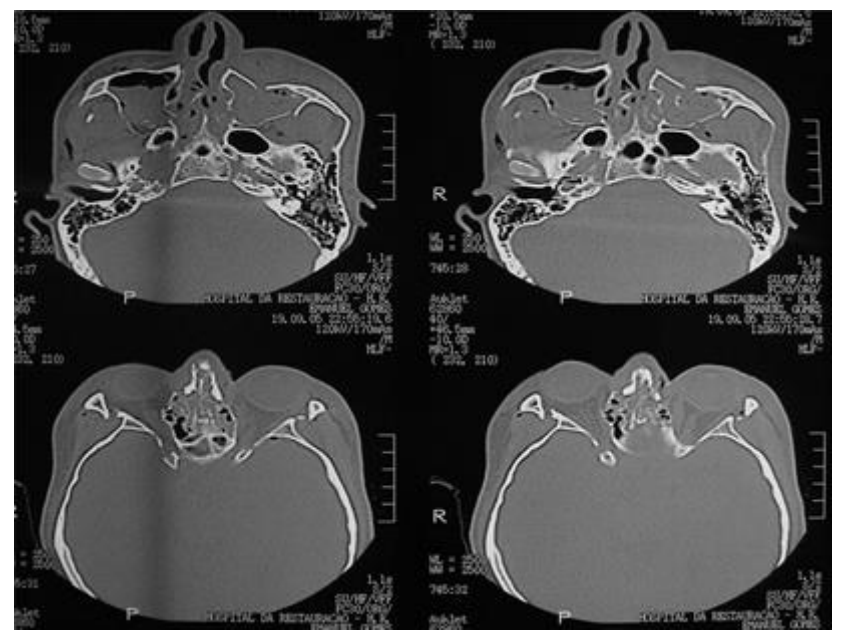

Figura 2: Imagens Axiais de Tomografia Computadorizada préoperatórias.

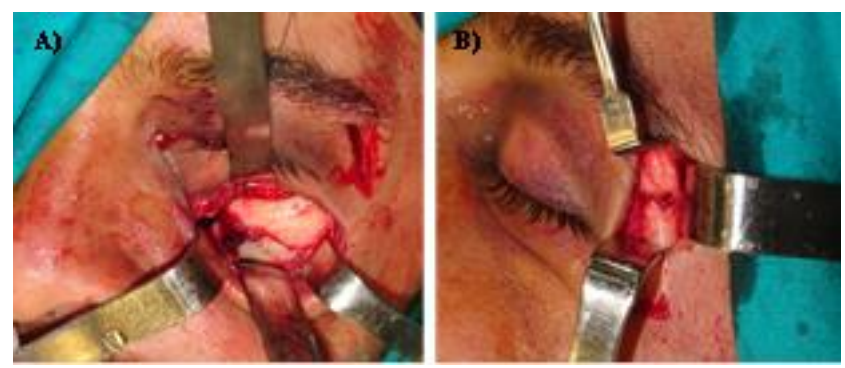

Figura 3: A) Acesso cirúrgico subtarsal; B) Acesso cirúrgico superciliar.

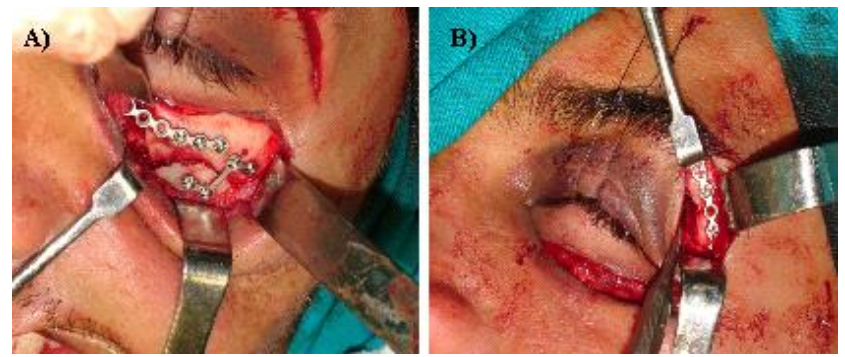

Figura 4: A) Osteossíntese com miniplaca e parafusos na região infraorbital E. B) Osteossíntese com mini-placa com parafusos na região frontozingomática $\mathrm{E}$.

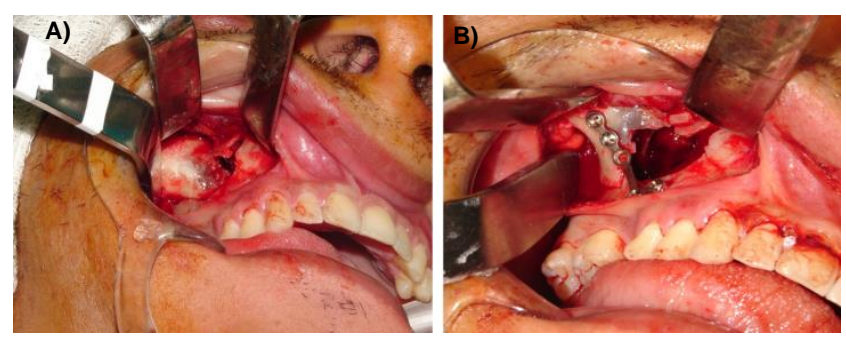

Figura 5: A) Acesso intra-oral do pilar zogomático-maxilar. B) Osteossíntese com placas e parafusos do pilar zigomático-maxilar.

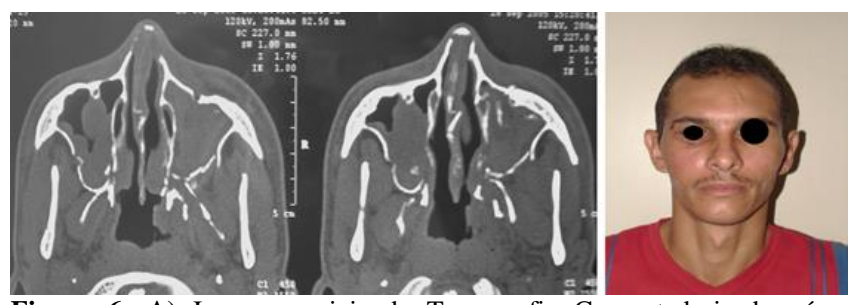

Figura 6: A) Imagens axiais de Tomografia Computadorizada pósoperatórias. B) Reestabelecimento da projeção anteroposterior do complexo zigomático-orbital.

\section{DISCUSSÃO}

O conhecimento da complexa relação anatômica e os meios de diagnóstico complementares são fundamentais para o sucesso do tratamento das fraturas do complexo zigomático. $\mathrm{O}$ plano de tratamento é determinado perante observação minuciosa das características clínicas presentes, como: dor no local da fratura, crepitação, alteração do sulco órbito-palpebral, equimose, limitação dos movimentos oculares, diplopia, parestesia infraorbitária e da pálpebra superior, hematoma e enoftalmia. A avaliação imaginológica também é importante, assim como o tipo de fratura, grau de fragmentação, a direção e o grau de deslocamento dos fragmentos ósseos ${ }^{9}$.

Os exames de imagens baseiam-se em solicitações de radiografias convencionais e tomografia computadorizada (TC), sendo as TC mais confiáveis, permitindo uma visão axial, sagital e coronal dos tecidos moles e duros ${ }^{10}$.

As fraturas do complexo zigomático representam um grande desafio ao cirurgião bucomaxilofacial, pois, o complexo zigomático é responsável pela dissipação de forças sobre o seu corpo e importante transmissor das cargas mastigatórias, sendo fundamental o seu restabelecimento anatômico na face ${ }^{11}$, com isso, o tratamento inadequado pode resultar em deformidades graves, implicações estéticas e funcionais significantes, e a correção cirúrgica secundária é geralmente de certa complexidade. A epistaxe, diplopia, equimose subconjuntival, exposição da esclera e diminuição da mobilidade, são consequências da má posição do osso fraturado ${ }^{7}$.

Não há um consenso quanto ao tratamento ideal das fraturas orbitárias na literatura. Alguns profissionais defendem a exploração sistemática de todas as paredes fraturadas e a sua respectiva reconstrução $^{12}$ enquanto outros, recomendam a realização da cirurgia apenas quando os pacientes passem a desenvolver sinais e sintomas usuais desta fratura, como diplopia e a enoftalmia, sem regressão no período de 14 dias. Ocorrendo o retrocesso destes sintomas e se estabelecendo função ocular satisfatória, outros pesquisadores alegam que pode instituir o tratamento conservador ${ }^{13}$.

A grande importância do tratamento cirúrgico é restabelecer a proteção ocular e devolver os contornos normais e a proeminência da face, devolvendo estética ${ }^{14}$. A via de acesso é variável, sendo os mais utilizados o subciliar, o infraorbitário e o transconjutival. Em alguns casos, acessa-se a órbita por feridas traumáticas com solução de continuidade. Outra forma de acesso utilizada é o transcaruncular, associado com o transconjuntival. Porém, cabe ao cirurgião escolher a técnica que melhor se adeque, 
pautado no melhor resultado pós-operatório e estético para o paciente ${ }^{15}$.

\section{CONCLUSÃO}

Neste caso, o diagnóstico precoce de fratura do complexo zigomático-orbital resultou em um melhor pós-operatório, reduzindo o risco de possíveis complicações estético-funcionais, além do restabelecimento anatômico, estético e funcional da região da fratura.

\section{REFERÊNCIAS}

1. Obimakinde OS, Ogundipe KO, Rabiu TB, Okoje VN. Maxillofacial fractures in a budding teaching hospital: a study of pattern of presentation and care. Pan Afr Med J. 2017;26:218.

2. Ugboko V, Udoye C, Ndukwe K, Amole A, Aregbesola S. Zygomatic complex fractures in a suburban Nigerian population. Dent Traumatol. 2005;21(2):70-5.

3. Zamboni RA, Wagner JCB, Volkweis MR, Gerhardt EL, Buchmann EM, Bavaresco CS. Epidemiological study of facial fractures at the Oral and Maxillofacial Surgery Service, Santa Casa de Misericordia Hospital Complex, Porto Alegre - RS - Brazil. Rev Col Bras Cir. 2017;44(5):491-97.

4. Ramos JC, Almeida MLD, Alencar YCG, de Sousa Filho LF, Figueiredo CHMC, Almeida MSC. Estudo epidemiológico do trauma bucomaxilofacial em um hospital de referência da Paraíba. Rev Col Bras Cir. 2018;45(6):e1978.

5. Wulkan M, Parreira Junior JG, Botter DA. Epidemiologia do trauma facial. Rev Assoc Med Bras. 2005;51(5):290-95.

6. Knight JS, North JF. The classification of malar fractures: an analysis of displacement as a guide to treatment. Br J Plast Surg. 1961;13:325-39.

7. Mendonça JCG, Crivelli DMB. Tratamento de fratura cominutiva do complexo zigomático orbitário com utilização de fio de aço: relato de caso. Rev Bras Cir Cabeça Pescoço. 2012; 41(2):93-5.

8. Hupp JR, Tucker MR, Ellis E.Cirurgia oral e maxilofacial contemporânea. 6. ed. Rio de Janeiro: Elsevier; 2015. p. 1099-1113.

9. Oliva MA. Acesso subciliar para fraturas do complexozigomático-orbitári. Rev Bras Cir Cabeça Pescoço. 2013;42(2):106-8.

10. Soares LP, Gaião L, Santos MESM, Pozza DH, Oliveira MG. Indicações da Tomografia Computadorizada no Diagnóstico das Fraturas Nasoórbito-etmoidais. Rev de Clin Pesq Odontol. 2004;1(1):29-33.

11. Kurita M, Okazaki M, Ozaki M, Tanaka Y, Tsuji N, Takushima A, et al. Patient satisfaction after open reduction and internal fixation of zygomatic bone fractures. J Craniofac Surg .2010;21(7):45-9.
12. Hammer B. Fraturas orbitárias: Diagnóstico, tratamento cirúrgico, correções secundárias. São Paulo: Santos; 2005.

13. Panarello F, Chaves Júnior AC, Leles JLR, Oliveira MG. Análise dos materiais empregados para a reconstrução das fraturas orbitárias Revisão de literatura. RBC: Rev Int Cir Traumatol Bucomaxilofacial. 2005;3(9):57-64.

14. Kloss FR, Stigler RG, Brandstätter A, Tuli T, Rasse M, Laimer K, Hächl OL, Gassner R. Complications related to midfacial fractures: operative versus non-surgical treatment. Int $\mathbf{J}$ Oral Maxillofac Surg. 2011;40(1):33-7.

15. Liedtke FS, Richinho KP, Pisanelli CH, Araf D. Fraturas do soalho da órbita do tipo "Blow-out": revisão de literatura. Revicience 2005;5(5):8-11.

\section{CONFLITO DE INTERESSES}

Os autores declaram não haver conflitos de interesse.

\section{AUTOR PARA CORRESPONDÊNCIA}

\section{Micaella Fernandes Farias}

Centro Universitário João Pessoa - Unipê 58053-000 João Pessoa - PB, Brasil

E-mail:micaellaff@gmail.com
Submetido em 20/02/2020 Aceito em 30/07/2020 